\title{
Inhibitory Effects of Triterpene-Azidothymidine Conjugates on Proliferation of Human Immunodeficiency Virus Type 1 and Its Protease
}

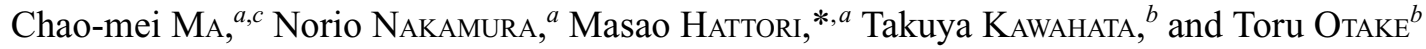 \\ ${ }^{a}$ Institute of Natural Medicine, Toyama Medical and Pharmaceutical University; 2630 Sugitani, Toyama 930-0194, Japan: \\ ${ }^{b}$ Osaka Prefectural Institute of Public Health; Osaka 537-0025, Japan: and ${ }^{c}$ School of Pharmaceutical Sciences, Peking \\ University; Beijing 100083, P. R. China. Received January 23, 2002; accepted March 8, 2002
}

The conjugates of some dicarboxylic acid hemiesters of triterpenes which show potent inhibition against human immunodeficiency virus type 1 protease (HIV-1 PR) with a reverse transcriptase inhibitor azidothymidine (AZT) or anti-HIV alkaloid FK 3000 were prepared, and their inhibitory activities were investigated against HIV-induced cytopathic effects (CPE) and HIV-1 PR. Most of the triterpene-AZT conjugates showed potent antiHIV activity as well as moderate to potent $P R$ inhibitory activity, though AZT itself showed no PR inhibitory activity at all. However, the triterpene-FK 3000 conjugates showed neither PR inhibitory activity nor anti-HIV activity.

Key words FK 3000; azidothymidine (AZT); protease inhibition; reverse transcriptase inhibition; triterpene-AZT conjugate; human immunodeficiency virus

Currently, two types of inhibitors of human immunodeficiency virus (HIV) have been developed as therapeutic drugs for acquired immunodeficiency syndrome (AIDS). One is an HIV-1 reverse transcriptase (RT) inhibitor, and the other is an HIV-1 protease (PR) inhibitor. Both enzymes are essential for the replication of HIV. Combinational uses of these two types of agents have succeeded in lowering the emergency of the viral resistance to anti-HIV drugs. However, the virus cannot be completely eradicated from the bodies of patients and these anti-HIV drugs are still too expensive to be used by a majority of AIDS patients. Many attempts to develop new anti-HIV agents have led to the synthesis and isolation of a variety of HIV-1 PR or RT inhibitors. However, many of the enzyme inhibitors showed poor anti-HIV activity regardless of their potent enzyme inhibitory activities. Recently, another concept of drug combination emerged, that is to link two types of HIV inhibitors with a chemical bond, such as conjugates of HIV-1 RT inhibitory nucleosides with nonnucleosides, ${ }^{1,2)}$ and of HIV-1 RT inhibitors with HIV-1 PR inhibitors. ${ }^{3)}$ The conjugates of two enzyme inhibitors are not only expected to lower the speed of the emergence of virusdrug resistance, ${ }^{1)}$ but also to enhance the anti-HIV activity. ${ }^{3}$

We have previously reported that dicarboxylic acid hemiesters of ursolic acid, oleanolic acid and betulinic acid showed potent inhibitory activity on HIV-1 PR. ${ }^{4,5)}$ However, most of these derivatives showed no appreciable anti-HIV activity, which was believed to be due to the poor cell permeability. Following the concept mentioned above, we prepared some conjugates of triterpene derivatives with other anti-HIV agents, and their anti-HIV activity and HIV-1 PR inhibitory activity were compared with those of the respective units in the present paper.

\section{Results and Discussion}

Compounds $\mathbf{2}-\mathbf{8}, \mathbf{1 a}-\mathbf{8 a}$ were synthesized as reported previously. ${ }^{5)}$ The triterpene-AZT (azidothymidine) conjugates $(\mathbf{1 b}, \mathbf{2 b})$ and triterpene-FK 3000 conjugates $(\mathbf{2 f}, \mathbf{4 f}, \mathbf{6 f})$ were synthesized by condensation of the corresponding dicarboxylic acid hemiesters (1a, 2a, 4a, 6a) with AZT or FK 3000 in the presence of dicyclohexylcarbodiimide (DCC) and 4-dimethylaminopyridine (DMAP) in $\mathrm{CH}_{2} \mathrm{Cl}_{2}-\mathrm{CH}_{3} \mathrm{CN}$.
Compounds $\mathbf{3 b}-\mathbf{8 b}$ were prepared by coupling the respective amines or oximes with adipic acid hemiester of AZT, the latter being prepared by condensation of AZT with adipic acid using a DCC-DMAP method.

The inhibitory activity of the synthesized triterpene conjugates against HIV-1 PR was evaluated by quantitative high performance liquid chromatographic (HPLC) analysis of the

Table 1. Anti-HIV Protease and Anti-HIV Activity of Triterpene Derivatives

\begin{tabular}{|c|c|c|c|c|c|}
\hline \multirow{2}{*}{ Compd. } & \multirow{2}{*}{$\begin{array}{c}v s . \mathrm{PR} \\
\mathrm{IC}_{50}(\mu \mathrm{M})\end{array}$} & \multicolumn{2}{|c|}{ vs. HIV } & \multirow[b]{2}{*}{ S.I. } & Relative potency \\
\hline & & $\mathrm{IC}_{100}(\mu \mathrm{M})$ & $\mathrm{CC}_{0}(\mu \mathrm{M})$ & & to $\mathrm{AZT}$ \\
\hline 1 & 8 & $\mathrm{NE}$ & $\geq 110$ & & \\
\hline 2 & 20 & $13.4^{a)}$ & $\geq 53.1$ & 3.97 & $1 / 223-1 / 464$ \\
\hline 3 & 5.5 & $\mathrm{NE}$ & 266 & & \\
\hline 4 & 9.5 & $\mathrm{NE}$ & 51.8 & & \\
\hline $5+7$ & & $\mathrm{NE}$ & 2198 & & \\
\hline 6 & $>20$ & $\mathrm{NE}$ & 13.3 & & \\
\hline 8 & $>20$ & $\mathrm{NE}$ & 13.3 & & \\
\hline 1a & 3.0 & $\mathrm{NE}$ & 13.4 & & \\
\hline $2 a$ & 7.5 & $20.9^{b)}$ & 41.8 & 2.00 & $1 / 349$ \\
\hline $3 \mathbf{a}$ & 5.5 & $\mathrm{NE}$ & 209 & & \\
\hline $4 a$ & 4.0 & $\mathrm{NE}$ & 40.9 & & \\
\hline $5 a$ & 3.0 & $\mathrm{NE}$ & 53.7 & & \\
\hline $6 a$ & 3.0 & $\mathrm{NE}$ & 419 & & \\
\hline $7 a$ & 2.1 & $\mathrm{NE}$ & 21.4 & & \\
\hline $8 \mathbf{a}$ & 3.5 & NE & 53.3 & & \\
\hline $1 b$ & 3.2 & $3.78^{a)}$ & 15.0 & 3.99 & $1 / 129-1 / 62.8$ \\
\hline $2 b$ & 4.0 & $18.4^{c)}$ & 148 & 8.01 & $1 / 632$ \\
\hline $3 \mathbf{b}$ & 1.9 & $1.84^{d)}$ & 29.6 & 16.1 & $1 / 30.7$ \\
\hline $4 b$ & 16 & $4.53^{c)}$ & 145 & 32.0 & $1 / 75.6$ \\
\hline $5 \mathbf{b}$ & 1.2 & $0.589^{d)}$ & 120 & 204 & $1 / 9.83$ \\
\hline $6 \mathbf{b}$ & 20 & $0.370^{c)}$ & 73.9 & 200 & $1 / 12.7$ \\
\hline $7 \mathbf{b}$ & 1.9 & $0.469^{d)}$ & 120 & 256 & $1 / 7.8$ \\
\hline $8 b$ & 8.0 & $7.39^{d)}$ & $>118$ & $>16.0$ & $1 / 123$ \\
\hline $2 f$ & $>100$ & $\mathrm{NE}$ & 502 & & \\
\hline $4 f$ & $>100$ & $\mathrm{NE}$ & 31.0 & & \\
\hline $6 f$ & $>100$ & $\mathrm{NE}$ & 1004 & & \\
\hline \multirow[t]{3}{*}{$\mathrm{AZT}$} & $>100$ & $0.0599-0.0291^{a)}$ & $>1^{b, c)}$ & $>62.5^{b)}$ & 1 \\
\hline & & $0.0599^{b, d)}$ & $1^{d)}$ & $>128.2^{c)}$ & \\
\hline & & $0.0291^{c)}$ & & $62.5^{d)}$ & \\
\hline
\end{tabular}

$\mathrm{IC}_{100}$, the minimum concentration for complete inhibition of HIV-1 induced CPE in MT- 4 cells by microscopic observation. $\mathrm{CC}_{0}$, the minimum concentration for appearance of MT-4 cell toxicity by microscopic observation. NE, not effective. $a-d$ ) Samples with their $\mathrm{IC}_{100}$ values bearing the same superscrip were measured at the same time. 
cleavage products of a synthetic substrate. The 50\% inhibitory concentrations $\left(\mathrm{IC}_{50}\right)$ of these conjugates against HIV-1 PR are listed in Table 1 . The anti-HIV activity and cytotoxicity of the conjugates were evaluated with MT-4 cells. The results were represented as $\mathrm{IC}_{100}$ and $\mathrm{CC}_{0}$ values, referring to the minimum concentration for complete inhibition of HIV-1 induced cytopathic effects (CPE) and the minimum concentration for appearance of MT-4 cell toxicity, respectively.

Although potent HIV-1 PR inhibitory activity was observed in many dicarboxylic acid hemiesters of oleanolic acid (1a-8a), most of them exhibited no appreciable antiHIV activity. It has been reported that some compounds with free carboxylic groups in their structures showed potent HIV1 PR inhibitory activity but little anti-HIV activity due to their poor cell permeability. ${ }^{3)}$ Conjugates of these HIV-1 PR inhibitors with a reverse transcriptase inhibitor, such as AZT, may increase their permeability to the cell. The conjugates then split into two different classes of anti-HIV agents inside the cell and thus express excellent antiviral activity. ${ }^{3)}$ Inspired by this concept, we prepared several triterpene-AZT conjugates and triterpene-FK 3000 conjugates. FK 3000 is a morphinane type alkaloid having anti-herpes simplex virus activity in vitro and in vivo by inhibiting viral DNA synthesis. $^{6-8)}$ In addition, we found that FK 3000 also had anti-HIV activity $\left(\mathrm{IC}_{100}=7.8 \mu \mathrm{g} / \mathrm{ml}, \mathrm{CC}_{0}=15.6 \mu \mathrm{g} / \mathrm{ml}\right)$ but no inhibi-

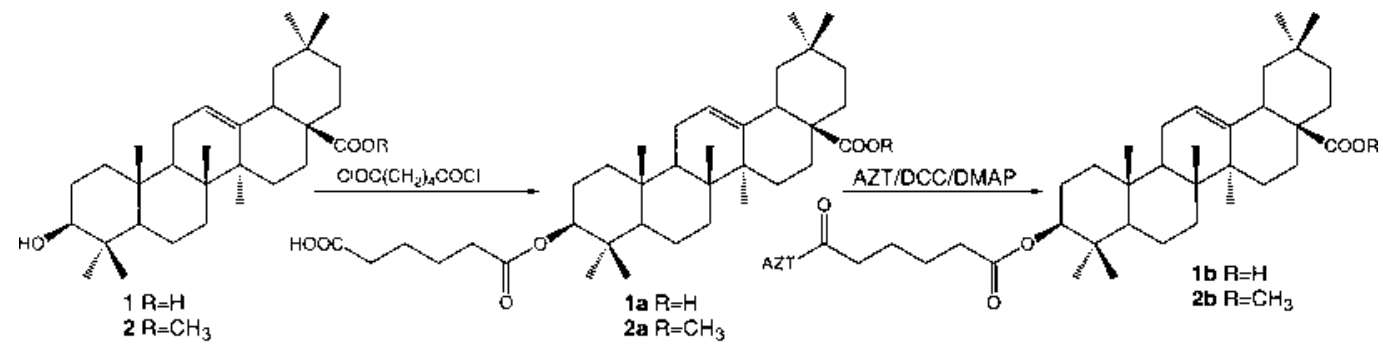

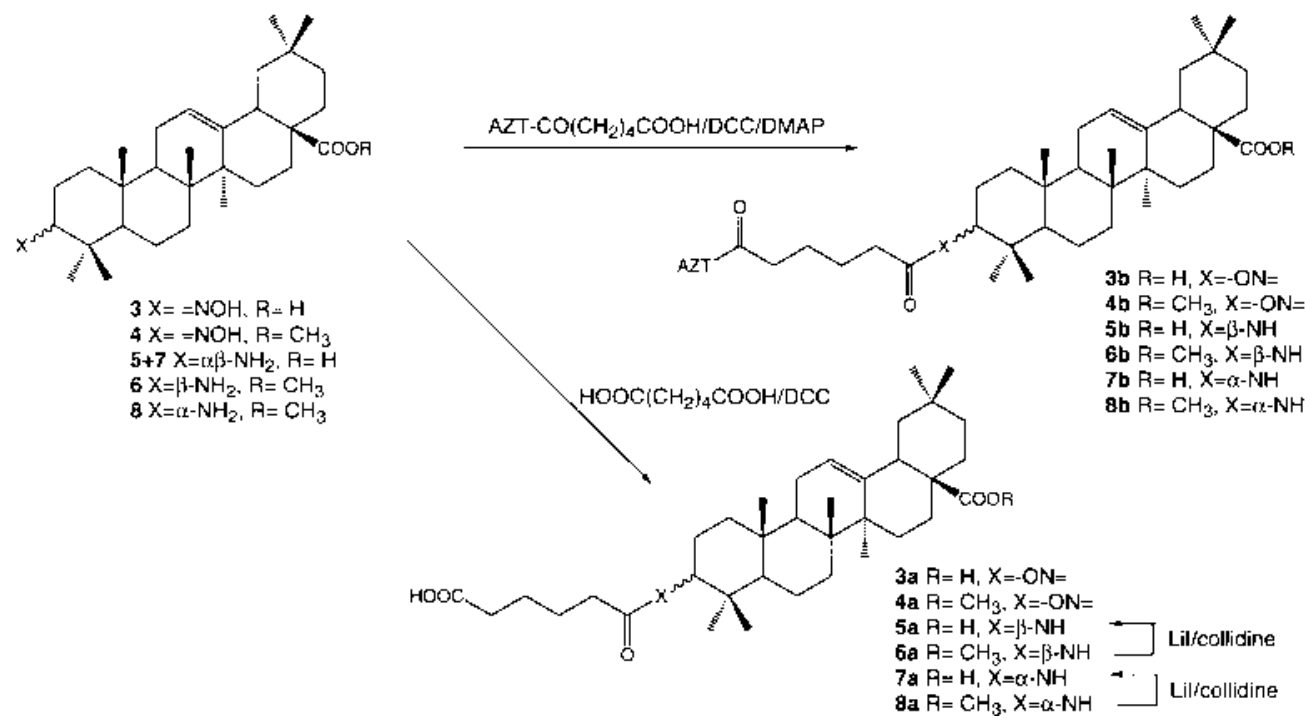

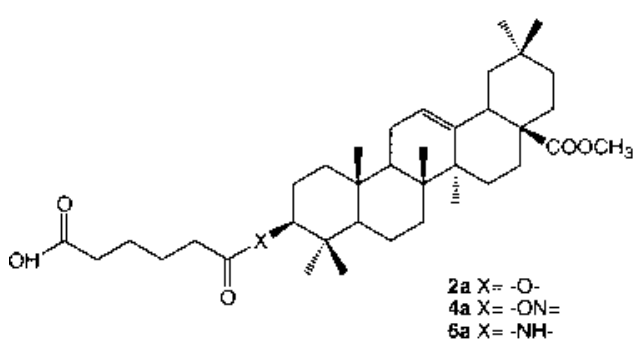

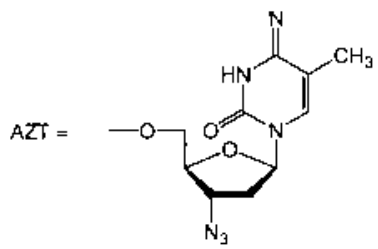

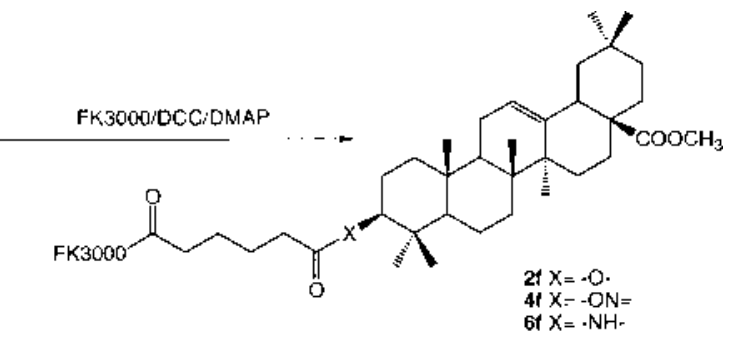

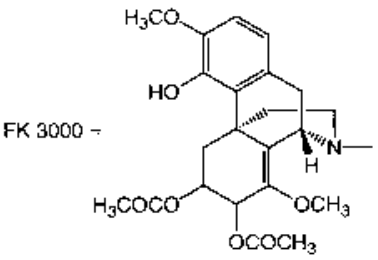

Fig. 1. Synthesis of Triterpene-AZT and Triterpene-FK 3000 Conjugates 
tory activity against HIV-1 PR, suggesting that it targets a stage of the viral life cycle, different from that mediated by PR. When all the conjugates were tested for their anti-HIV activity and PR inhibitory activity, the triterpene-FK 3000 conjugates (2f, 4f, 6f) showed neither inhibitory activity against HIV-1 PR nor anti-HIV activity. The poor anti-HIV activity of the triterpene-FK 3000 conjugates may be due to the less permeability to cells and/or the difficulty in cleaving an amide bond of the conjugates into two active inhibitory components inside the cells.

On the other hand, all the triterpene-AZT conjugates (1b-8b) showed potent anti-HIV activity (Table 1) and this activity was compared with the relative potency of samples to AZT. The 28-free acid derivatives $(\mathbf{1 b}, \mathbf{3 b}, \mathbf{5 b}, \mathbf{7 b})$ were more potent than the corresponding 28-methyl ester derivatives $(\mathbf{2} \mathbf{b}, \mathbf{4 b}, \mathbf{6 b}, \mathbf{8 b})$. With respect to the chemical bond at position-3, the 3 -amide conjugates $(\mathbf{5 b}-\mathbf{7 b}$ except for $\mathbf{8 b})$ were the most potent followed by the 3 -imide conjugates $(\mathbf{3 b}$, 4b). The conjugates with a 3 -ester bond $(\mathbf{1 b}, \mathbf{2 b})$ showed the weakest anti-HIV activity among the triterpene-AZT conjugates (1b-8b). The most potent compound (7b) completely inhibited HIV-1 induced CPE on MT-4 cells at $0.47 \mu \mathrm{M}, 1 / 7.8$ fold of the potency of AZT.

Although AZT itself showed no inhibitory activity against HIV-1 PR, most of the triterpene-AZT conjugates exhibited moderate to potent PR inhibitory activity. Especially, those had a free acid group at position-28 (1b, 3b, 5b, 7b) showed inhibitory activity against HIV-1 PR comparable to or even more potent than the corresponding triterpene dicarboxylic acid hemiesters (1a, 3a, 5a, 7a). The most potent conjugate 5b had an $\mathrm{IC}_{50}$ as low as $1.2 \mu \mathrm{M}$. Since no hydrolysis of the triterpene-AZT conjugates was observed under the assay conditions when monitored by HPLC, the triterpene-AZT conjugates but not the hydrolysates were responsible for the inhibitory activity of $\mathbf{1 b}-\mathbf{8 b}$ against HIV-1 PR. Size exclusion chromatography of $\mathbf{5 b}$ using the reported method ${ }^{5)}$ revealed that the action mechanism was dimerization inhibition (data not shown). The improved potency of triterpene-AZT conjugates over triterpene dicarboxylic acid hemiesters may indicate that the overall structures of the triterpene conjugate could fit at a site of HIV-1 PR leading to the inhibition of dimerization. Although the anti-HIV activity of these conjugates was weaker than that of AZT itself, the PR inhibitory effects may be effective for AZT-resistant virus.

\section{Experimental}

General Experimental Procedures Optical rotations were measured with a JASCO DIP-360 automatic polarimeter. Proton nuclear magnetic resonance ( $\left.{ }^{1} \mathrm{H}-\mathrm{NMR}\right)$ spectra were measured with either a Varian Gemini 300 $\left({ }^{1} \mathrm{H}, 300 \mathrm{MHz}\right)$, Varian Unity $500\left({ }^{1} \mathrm{H}, 500 \mathrm{MHz}\right)$ or JEOL JNA-LA 400WBFT $\left({ }^{1} \mathrm{H}, 400 \mathrm{MHz}\right)$ NMR spectrometer. The chemical shifts are represented in ppm with tetramethylsilane (TMS) as an internal standard. Atmospheric pressure ionization mass (API-MS) spectra were measured with a PerkinElmer SCIEX API-III biomolecular mass analyzer. Preparative HPLC was carried out on a Gilson instrument with a 231 LX injector, a 119 UV/VIS detector and a TSK gel ODS- $80 \mathrm{~T}_{\mathrm{M}}$ column $(21.5 \times 300 \mathrm{~mm}$, Tosoh Co. $)$.

Chemicals and Enzyme FK 3000 was isolated from the root tubers of Stephania cepharantha HAYATA as reported previously. ${ }^{6}$ An HIV-protease assay kit was purchased from Backem Bioscience.

General Procedure for Coupling of Oleanolic Acid Derivatives with AZT (1b, 2b) Azidothymidine $(70 \mathrm{mg}, 0.26 \mathrm{mmol})$ and DCC $(8.0 \mathrm{mg}$, $0.04 \mathrm{mmol}$ ) were added to a solution of triterpene acid (1a or $\mathbf{2 a} 0.02-$ $0.04 \mathrm{mmol})$ and DMAP $(4.0 \mathrm{mg}, 0.03 \mathrm{mmol})$ in a mixture of $\mathrm{CH}_{2} \mathrm{Cl}_{2}-$ $\mathrm{CH}_{3} \mathrm{CN}(4: 1,2 \mathrm{ml})$. The reaction mixture was allowed to stand for $10 \mathrm{~h}$ at room temperature with stirring. The solvent was then removed, and the residue was subjected to column chromatography on $\mathrm{RP}-18\left(\mathrm{H}_{2} \mathrm{O}-\mathrm{MeOH}\right.$ $3: 7-0: 1)$.

3-O-[6-O-(Azidothymidine-5'-yl)-adipoyl]oleanolic Acid (1b): 33.7\%, $[\alpha]_{\mathrm{D}}^{25}+46.3^{\circ}\left(c=0.22, \mathrm{CHCl}_{3}\right) ;{ }^{1} \mathrm{H}-\mathrm{NMR}\left(\mathrm{CDCl}_{3}, 400 \mathrm{MHz}\right) \delta: 0.75(3 \mathrm{H}$, s), $0.84(3 \mathrm{H}, \mathrm{s}), 0.85(3 \mathrm{H}, \mathrm{s}), 0.90(3 \mathrm{H}, \mathrm{s}), 0.93(6 \mathrm{H}, \mathrm{s}), 1.13(3 \mathrm{H}, \mathrm{s})$ $\left(7 \times \mathrm{CH}_{3}\right), 1.93\left(3 \mathrm{H}, \mathrm{d}, J=1.0 \mathrm{~Hz}, \mathrm{CH}_{3}-\mathrm{Nu}\right), 2.34\left(2 \mathrm{H}, \mathrm{t}\right.$-like, $\left.\mathrm{H}-2^{\prime}\right), 2.41$ (2H, t-like, H-5') 2.46 (2H, m, H-2'-Nu), $2.83(1 \mathrm{H}, \mathrm{dd}, J=13.5,4.1 \mathrm{~Hz}, \mathrm{H}-$ 18), $4.06\left(1 \mathrm{H}, \mathrm{q}, J=4.6 \mathrm{~Hz}, \mathrm{H}-4^{\prime}-\mathrm{Nu}\right), 4.22\left(1 \mathrm{H}, \mathrm{dt}, J=7.5,5.5 \mathrm{~Hz}, \mathrm{H}-3^{\prime}-\right.$ $\mathrm{Nu}), 4.31\left(1 \mathrm{H}, \mathrm{dd}, J=3.8,12.2 \mathrm{~Hz}, \mathrm{Ha}-5^{\prime}-\mathrm{Nu}\right), 4.38(1 \mathrm{H}, \mathrm{dd}, J=4.6,12.1 \mathrm{~Hz}$, Hb-5'-Nu), $4.50(1 \mathrm{H}, \mathrm{t}, J=7.8 \mathrm{~Hz}, \mathrm{H}-3), 5.27(1 \mathrm{H}, \mathrm{t}-\mathrm{like}, \mathrm{H}-12), 6.09(1 \mathrm{H}, \mathrm{t}$, $\left.J=6.3 \mathrm{~Hz}, \mathrm{H}-1^{\prime}-\mathrm{Nu}\right), 7.21(1 \mathrm{H}, \mathrm{d}, J=1.2 \mathrm{~Hz}, \mathrm{H}-6-\mathrm{Nu}), 9.36(1 \mathrm{H}, \mathrm{br} \mathrm{s}, \mathrm{NH}-$ $\mathrm{Nu}$ ); API-MS (positive): $\mathrm{m} / \mathrm{z} 856[\mathrm{M}+\mathrm{Na}]^{+}(100)$; (negative): $832[\mathrm{M}-1]^{-}$ (100), 597 (50), 583 (70), 455 (35).

3-O-[6-O-(Azidothymidine-5'-yl)adipoyl]oleanolic Acid Methyl Ester (2b): $28.9 \%,[\alpha]_{\mathrm{D}}^{25}+47.2^{\circ}\left(c=0.72, \mathrm{CHCl}_{3}\right) ;{ }^{1} \mathrm{H}-\mathrm{NMR}\left(\mathrm{CDCl}_{3}\right) \delta: 0.72(3 \mathrm{H}$, s), $0.85(6 \mathrm{H}, \mathrm{s}), 0.90(3 \mathrm{H}, \mathrm{s}), 0.93(6 \mathrm{H}, \mathrm{s}), 1.13(3 \mathrm{H}, \mathrm{s})\left(7 \times \mathrm{CH}_{3}\right), 1.65$ (overlapped signals, H-3', 4') $1.93\left(3 \mathrm{H}, \mathrm{d}, J=0.9 \mathrm{~Hz}, \mathrm{CH}_{3}-\mathrm{Nu}\right), 2.33(2 \mathrm{H}, \mathrm{t}$, $\left.J=6.9 \mathrm{~Hz}, \mathrm{H}-2^{\prime}\right), 2.41\left(2 \mathrm{H}, \mathrm{t}, J=6.5 \mathrm{~Hz}, \mathrm{H}-5^{\prime}\right), 2.44\left(2 \mathrm{H}, \mathrm{m}, \mathrm{H}-2^{\prime}-\mathrm{Nu}\right), 2.87$ $(1 \mathrm{H}, \mathrm{dd}, J=13.5,4.1 \mathrm{~Hz}, \mathrm{H}-18), 3.62\left(3 \mathrm{H}, \mathrm{s}, \mathrm{COOCH}_{3}-28\right), 4.06(1 \mathrm{H}, \mathrm{q}$, $\left.J=4.6 \mathrm{~Hz}, \mathrm{H}-4^{\prime}-\mathrm{Nu}\right), 4.21\left(1 \mathrm{H}, \mathrm{dt}, J=7.5,5.4 \mathrm{~Hz}, \mathrm{H}-3^{\prime}-\mathrm{Nu}\right), 4.31(1 \mathrm{H}, \mathrm{dd}$, $\left.J=3.9,12.0 \mathrm{~Hz}, \mathrm{Ha}-5^{\prime}-\mathrm{Nu}\right), 4.38\left(1 \mathrm{H}, \mathrm{dd}, J=4.3,12.0 \mathrm{~Hz}, \mathrm{Hb}-5^{\prime}-\mathrm{Nu}\right), 4.50$ $(1 \mathrm{H}, \mathrm{t}, J=7.8 \mathrm{~Hz}, \mathrm{H}-3), 5.28(1 \mathrm{H}, \mathrm{t}-\mathrm{like}, \mathrm{H}-12), 6.10\left(1 \mathrm{H}, \mathrm{t}, J=6.3 \mathrm{~Hz}, \mathrm{H}-1^{\prime}-\right.$ $\mathrm{Nu}), 7.20(1 \mathrm{H}, \mathrm{d}, J=0.9 \mathrm{~Hz}, \mathrm{H}-6-\mathrm{Nu}), 8.49(1 \mathrm{H}, \mathrm{brs}$, NH-Nu$)$; API-MS (negative): $m / z 846[\mathrm{M}-1]^{-}(55), 455$ (20).

Adipoylazidothymidine A mixture of azidothymidine $(300 \mathrm{mg}, 1.11$ $\mathrm{mmol}$ ), adipic acid ( $800 \mathrm{mg}, 4.44 \mathrm{mmol})$, 4-dimethylaminopyridine (DMAP) $(80 \mathrm{mg}, 0.65 \mathrm{mmol})$ and DCC $(300 \mathrm{mg}, 1.45 \mathrm{mmol})$ in tetrahydrofuran (THF, $50 \mathrm{ml}$ ) was stirred overnight at room temperature. The solution was then concentrated to dryness under vacuum and purified by ODS column chromatography $\left(\mathrm{H}_{2} \mathrm{O}-\mathrm{MeOH} 100: 0-40: 60\right)$ to give adipoylazidothymidine ( $320 \mathrm{mg}, 73.0 \%$ yield) as a white powder; API-MS (negative): $\mathrm{m} / \mathrm{z} 394$ $[\mathrm{M}-1]^{+}(80)$.

$3 \alpha$ - and $3 \beta$-Amino-olean-12-en-28-olic Acids $(5,7)$ Sodium cyanoborohydride $(0.42 \mathrm{~g}, 9 \mathrm{mmol})$ was added to a methanol solution of 3-hydroxyimino-olean-12-en-28-oic acid $(200 \mathrm{mg}, 0.41 \mathrm{mmol})^{5}$ ) and ammonium acetate $(0.37 \mathrm{~g}, 6.8 \mathrm{mmol})$ under argon atmosphere. The solution was cooled in ice water, and $15 \%$ aqueous titanium bichloride $(1.2 \mathrm{ml}, 1.4 \mathrm{mmol})$ was added dropwise over $20 \mathrm{~min}$. The mixture was stirred at room temperature for $12 \mathrm{~h}$ and then treated with $2 \mathrm{~N}$ sodium hydroxide to adjust to $\mathrm{pH} 10$. The aqueous solution was extracted with $\mathrm{CHCl}_{3}(150 \mathrm{ml} \times 2)$, and the organic layer was repeatedly washed with water and concentrated to dryness to give a mixture of $3 \alpha$ - and $3 \beta$-amino compounds as a white powder, in a yield of $89.0 \%$

Preparation of Compounds $3 \mathbf{b}-\mathbf{8 b}$ A mixture of adipoylazidothymidine $(27-60 \mathrm{mg}, 0.07 \mathrm{mmol}), \mathbf{3}, \mathbf{4}, \mathbf{6}, \mathbf{8}$, or $3 \alpha$ - and $3 \beta$-amino-oleanolic acids $(5+7)(15-30 \mathrm{mg}, 0.03-0.06 \mathrm{mmol})$, DMAP $(7 \mathrm{mg}, 0.06 \mathrm{mmol})$ and DCC $(16 \mathrm{mg}, 0.08 \mathrm{mmol})$ in THF $(2 \mathrm{ml})$ was stirred overnight at room temperature until the starting material disappeared. The reaction mixture was then treated with water, concentrated to dryness under vacuum, and purified by chromatography on a column of ODS and HPLC $\left(\mathrm{H}_{2} \mathrm{O}-\mathrm{MeOH} 20: 80-\right.$ $0: 100)$ to give compounds $\mathbf{3 b}-\mathbf{8 b}$.

Compound 3b: $54.4 \%$ in yield, $[\alpha]_{\mathrm{D}}^{25}+39.5^{\circ}\left(c=1.22, \mathrm{CHCl}_{3}\right) ;{ }^{1} \mathrm{H}-\mathrm{NMR}$ $\left(\mathrm{CDCl}_{3}\right) \delta: 0.77(3 \mathrm{H}, \mathrm{s}), 0.89(3 \mathrm{H}, \mathrm{s}), 0.92(3 \mathrm{H}, \mathrm{s}), 0.99(3 \mathrm{H}, \mathrm{s}), 1.11(6 \mathrm{H}$, s), $1.24(3 \mathrm{H}, \mathrm{s})\left(7 \times \mathrm{CH}_{3}\right), 1.93\left(3 \mathrm{H}, \mathrm{br} \mathrm{s}, \mathrm{CH}_{3}-\mathrm{Nu}\right), 2.42(7 \mathrm{H}$, overlapped signals, H-2'-Nu, Ha-2, 2' $\left.{ }^{\prime} 5^{\prime}\right), 2.85$ ( $2 \mathrm{H}$, overlapped signals, H-18, Hb-2), 4.06 $\left(1 \mathrm{H}, \mathrm{q}, J=4.5 \mathrm{~Hz}, \mathrm{H}-4^{\prime}-\mathrm{Nu}\right), 4.24\left(1 \mathrm{H}, \mathrm{dt}, J=7.2,5.4 \mathrm{~Hz}, \mathrm{H}-3^{\prime}-\mathrm{Nu}\right), 4.30$ $\left(1 \mathrm{H}, \mathrm{dd}, J=3.9,12.0 \mathrm{~Hz}, \mathrm{Ha}-5^{\prime}-\mathrm{Nu}\right), 4.38\left(1 \mathrm{H}, \mathrm{dd}, J=4.5,12.3 \mathrm{~Hz}, \mathrm{Hb}-5^{\prime}-\right.$ $\mathrm{Nu}), 5.27(1 \mathrm{H}, \mathrm{t}-\mathrm{like}, \mathrm{H}-12), 6.09\left(1 \mathrm{H}, \mathrm{t}, J=6.3 \mathrm{~Hz}, \mathrm{H}-1^{\prime}-\mathrm{Nu}\right), 7.20(1 \mathrm{H}$, br s, H-6-Nu); API-MS (negative): $m / z \quad 845$ [M-1] $^{-}$(80); Anal. Calcd for $\mathrm{C}_{46} \mathrm{H}_{66} \mathrm{O}_{9} \mathrm{~N}_{6}$ : C, 65.21; H, 7.86; N, 9.93. Found: C, 65.34; H, 7.98; N, 9.72.

Compound $4 \mathbf{b}: 58.1 \%$ in yield, $[\alpha]_{\mathrm{D}}^{25}+65.7^{\circ}\left(c=0.46, \mathrm{CHCl}_{3}\right) ;{ }^{1} \mathrm{H}-\mathrm{NMR}$ $\left(\mathrm{CDCl}_{3}\right) \delta: 0.76(3 \mathrm{H}, \mathrm{s}), 0.90(3 \mathrm{H}, \mathrm{s}), 0.93(3 \mathrm{H}, \mathrm{s}), 1.02(3 \mathrm{H}, \mathrm{s}), 1.12(3 \mathrm{H}$, s), $1.13(3 \mathrm{H}, \mathrm{s}), 1.25(3 \mathrm{H}, \mathrm{s})\left(7 \times \mathrm{CH}_{3}\right), 1.65$ (overlapped signals, $\left.\mathrm{H}-3^{\prime}, 4^{\prime}\right)$, $1.94\left(3 \mathrm{H}, \mathrm{d}, J=0.9 \mathrm{~Hz}, \mathrm{CH}_{3}-\mathrm{Nu}\right), 2.33\left(7 \mathrm{H}\right.$, overlapped signals, $\mathrm{H}-2^{\prime}-\mathrm{Nu}, \mathrm{H}-$ 2a, 2', 5') $2.89(2 \mathrm{H}$, overlapped signals, H-18, H-2b), $3.63(3 \mathrm{H}, \mathrm{s}$, $\left.\mathrm{COOCH}_{3}-28\right), 4.06\left(1 \mathrm{H}, \mathrm{q}, J=4.5 \mathrm{~Hz}, \mathrm{H}-4^{\prime}-\mathrm{Nu}\right), 4.23(1 \mathrm{H}, \mathrm{dt}, J=7.2$, $\left.5.4 \mathrm{~Hz}, \mathrm{H}-3^{\prime}-\mathrm{Nu}\right), 4.30\left(1 \mathrm{H}, \mathrm{dd}, J=3.9,12.0 \mathrm{~Hz}, \mathrm{Ha}-5^{\prime}-\mathrm{Nu}\right), 4.39(1 \mathrm{H}, \mathrm{dd}$, $\left.J=4.5,12.3 \mathrm{~Hz}, \mathrm{Hb}-5^{\prime}-\mathrm{Nu}\right), 5.29(1 \mathrm{H}, \mathrm{t}-\mathrm{like}, \mathrm{H}-12), 6.10(1 \mathrm{H}, \mathrm{t}, J=6.3 \mathrm{~Hz}$, $\left.\mathrm{H}-1^{\prime}-\mathrm{Nu}\right), 7.20(1 \mathrm{H}, \mathrm{d}, J=0.9 \mathrm{~Hz}, \mathrm{H}-6-\mathrm{Nu}), 8.52(1 \mathrm{H}, \mathrm{brs}$, NH-Nu); APIMS (negative): $m / z 859[\mathrm{M}-1]^{-}$(80).

Compound 5b: $58.4 \%$ in yield, $[\alpha]_{\mathrm{D}}^{25}+41.8^{\circ}\left(c=1.36, \mathrm{CHCl}_{3}\right) ;{ }^{1} \mathrm{H}-\mathrm{NMR}$ $\left(\mathrm{CDCl}_{3}-\mathrm{CD}_{3} \mathrm{OD}\right) \delta: 0.77(3 \mathrm{H}, \mathrm{s}), 0.78(3 \mathrm{H}, \mathrm{s}), 0.86(3 \mathrm{H}, \mathrm{s}), 0.90(6 \mathrm{H}, \mathrm{s})$, $0.93(3 \mathrm{H}, \mathrm{s}), 1.15(3 \mathrm{H}, \mathrm{s})\left(7 \times \mathrm{CH}_{3}\right), 1.92\left(3 \mathrm{H}, \mathrm{s}, \mathrm{CH}_{3}-\mathrm{Nu}\right), 2.21(2 \mathrm{H}, \mathrm{t}-\mathrm{like}$, $\left.\mathrm{H}-2^{\prime}\right), 2.43\left(4 \mathrm{H}\right.$, overlapped signals, $\left.\mathrm{H}-5^{\prime}, \mathrm{H}-2^{\prime}-\mathrm{Nu}\right), 2.82(1 \mathrm{H}, \mathrm{dd}, J=13.5$, 
$4.1 \mathrm{~Hz}, \mathrm{H}-18$ ), 3.65 (overlapped with $\mathrm{H}_{2} \mathrm{O}$ signal, $\left.\mathrm{H}-3\right), 4.06(1 \mathrm{H}, \mathrm{q}$, $\left.J=4.4 \mathrm{~Hz}, \mathrm{H}-4^{\prime}-\mathrm{Nu}\right), 4.25\left(1 \mathrm{H}, \mathrm{dt}, J=7.4,5.5 \mathrm{~Hz}, \mathrm{H}-3^{\prime}-\mathrm{Nu}\right), 4.30(1 \mathrm{H}, \mathrm{dd}$, $\left.J=3.8,12.1 \mathrm{~Hz}, \mathrm{Ha}-5^{\prime}-\mathrm{Nu}\right), 4.37\left(1 \mathrm{H}, \mathrm{dd}, J=4.4,12.1 \mathrm{~Hz}, \mathrm{Hb}-5^{\prime}-\mathrm{Nu}\right), 5.27$ $(1 \mathrm{H}, \mathrm{t}$-like, $\mathrm{H}-12), 6.08\left(2 \mathrm{H}\right.$, overlapped signals, $\left.\mathrm{NH}, \mathrm{H}-1^{\prime}-\mathrm{Nu}\right), 7.25(1 \mathrm{H}$, br s, H-6-Nu); API-MS (negative): $m / z 831$ [M-1] $^{-}$(100); Anal. Calcd for $\mathrm{C}_{46} \mathrm{H}_{68} \mathrm{O}_{8} \mathrm{~N}_{6}: \mathrm{C}, 66.31 ; \mathrm{H}, 8.23 ; \mathrm{N}, 10.09$. Found: C, 65.99; H, 8.30; N, 9.86.

Compound 6b: $70.9 \%$ in yield, $[\alpha]_{\mathrm{D}}^{25}+41.8^{\circ}\left(c=1.36, \mathrm{CHCl}_{3}\right) ;{ }^{1} \mathrm{H}-\mathrm{NMR}$ $\left(\mathrm{CDCl}_{3}\right) \delta: 0.72(3 \mathrm{H}, \mathrm{s}), 0.76(3 \mathrm{H}, \mathrm{s}), 0.88(3 \mathrm{H}, \mathrm{s}), 0.89(6 \mathrm{H}, \mathrm{s}), 0.90(3 \mathrm{H}$, s), $0.92(3 \mathrm{H}, \mathrm{s})\left(7 \times \mathrm{CH}_{3}\right), 1.65$ (overlapped signals, $\left.\mathrm{H}-3^{\prime}, 4^{\prime}\right), 1.93(3 \mathrm{H}, \mathrm{s}$, $\left.\mathrm{CH}_{3}-\mathrm{Nu}\right), 2.20(2 \mathrm{H}$, t-like, H-2'), 2.43 (4H, overlapped signals, H-5', H-2'$\mathrm{Nu}), 2.87(1 \mathrm{H}, \mathrm{dd}, J=13.5,4.1 \mathrm{~Hz}, \mathrm{H}-18), 3.62\left(3 \mathrm{H}, \mathrm{s}, \mathrm{COOCH}_{3}-28\right), 3.65$ (overlapped signals, H-3), $4.05\left(1 \mathrm{H}, \mathrm{q}, J=4.4 \mathrm{~Hz}, \mathrm{H}-4^{\prime}-\mathrm{Nu}\right), 4.23(1 \mathrm{H}, \mathrm{dt}$, $\left.J=7.4,5.5 \mathrm{~Hz}, \mathrm{H}-3^{\prime}-\mathrm{Nu}\right), 4.29\left(1 \mathrm{H}, \mathrm{dd}, J=3.8,12.1 \mathrm{~Hz}, \mathrm{Ha}-5^{\prime}-\mathrm{Nu}\right), 4.36$ $\left(1 \mathrm{H}, \mathrm{dd}, J=4.4,12.1 \mathrm{~Hz}, \mathrm{Hb}-5^{\prime}-\mathrm{Nu}\right), 5.28(1 \mathrm{H}, \mathrm{t}-\mathrm{like}, \mathrm{H}-12), 5.29(1 \mathrm{H}, \mathrm{d}$, $J=9.9 \mathrm{~Hz}, \mathrm{NH}), 6.08\left(1 \mathrm{H}, \mathrm{t}, J=6.3 \mathrm{~Hz}, \mathrm{H}-1^{\prime}-\mathrm{Nu}\right), 7.21(1 \mathrm{H}, \mathrm{br}, \mathrm{H}-6-\mathrm{Nu})$, $8.77\left(1 \mathrm{H}, \mathrm{br} \mathrm{s}\right.$, NH-Nu); API-MS (negative): $m / z 845[\mathrm{M}-1]^{-}(100)$.

Compound $7 \mathbf{b}: 16.7 \%$ in yield, $[\alpha]_{\mathrm{D}}^{25}+35.0^{\circ}\left(c=0.54, \mathrm{CHCl}_{3}\right) ;{ }^{1} \mathrm{H}-\mathrm{NMR}$ $\left(\mathrm{CDCl}_{3}\right) \delta: 0.76(3 \mathrm{H}, \mathrm{s}), 0.83(3 \mathrm{H}, \mathrm{s}), 0.91(3 \mathrm{H}, \mathrm{s}), 0.93(6 \mathrm{H}, \mathrm{s}), 1.18(3 \mathrm{H}, \mathrm{s})$ $\left(7 \times \mathrm{CH}_{3}\right), 1.93\left(3 \mathrm{H}, \mathrm{s}, \mathrm{CH}_{3}-\mathrm{Nu}\right), 2.26\left(2 \mathrm{H}, \mathrm{t}-\mathrm{like}, \mathrm{H}-2^{\prime}\right), 2.45(4 \mathrm{H}$, overlapped signals, $\left.\mathrm{H}-5^{\prime}, \mathrm{H}-2^{\prime}-\mathrm{Nu}\right), 2.83(1 \mathrm{H}, \mathrm{dd}, J=13.5,4.1 \mathrm{~Hz}, \mathrm{H}-18), 3.82$ $(1 \mathrm{H}$, br d, $J=9.3 \mathrm{~Hz}, \mathrm{H}-3), 4.06\left(1 \mathrm{H}, \mathrm{q}, J=4.4 \mathrm{~Hz}, \mathrm{H}-4^{\prime}-\mathrm{Nu}\right), 4.26(1 \mathrm{H}, \mathrm{dt}$, $\left.J=7.4,5.5 \mathrm{~Hz}, \mathrm{H}-3^{\prime}-\mathrm{Nu}\right), 4.34\left(2 \mathrm{H}, \mathrm{m}, \mathrm{H}-5^{\prime}-\mathrm{Nu}\right), 5.29(1 \mathrm{H}, \mathrm{t}-\mathrm{like}, \mathrm{H}-12)$, $5.78(1 \mathrm{H}, \mathrm{d}, J=9.3 \mathrm{~Hz}, \mathrm{NH}), 6.08\left(1 \mathrm{H}, \mathrm{t}, J=6.3 \mathrm{~Hz}, \mathrm{H}-1^{\prime}-\mathrm{Nu}\right), 7.22(1 \mathrm{H}$, br s, H-6-Nu), 8.94 (1H, br s, NH-Nu); API-MS (negative): $m / z 831[\mathrm{M}-1]^{-}$ (100).

Compound 8b: $68.3 \%$ in yield, $[\alpha]_{\mathrm{D}}^{25}+41.8^{\circ}\left(c=1.36, \mathrm{CHCl}_{3}\right) ;{ }^{1} \mathrm{H}-\mathrm{NMR}$ $\left(\mathrm{CDCl}_{3}\right) \delta: 0.73(3 \mathrm{H}, \mathrm{s}), 0.84(3 \mathrm{H}, \mathrm{s}), 0.91(3 \mathrm{H}, \mathrm{s}), 0.93(3 \mathrm{H}, \mathrm{s}), 0.95(6 \mathrm{H}$, s), $1.18(3 \mathrm{H}, \mathrm{s})\left(7 \times \mathrm{CH}_{3}\right), 1.93\left(3 \mathrm{H}, \mathrm{s}, \mathrm{CH}_{3}-\mathrm{Nu}\right), 2.24\left(2 \mathrm{H}, \mathrm{t}\right.$-like, $\left.\mathrm{H}-2^{\prime}\right), 2.43$ $(4 \mathrm{H}$, overlapped signals, H-5', H-2'-Nu), $2.87(1 \mathrm{H}, \mathrm{dd}, J=13.5,4.1 \mathrm{~Hz}, \mathrm{H}-$ 18), $3.63\left(3 \mathrm{H}, \mathrm{s}, \mathrm{COOCH}_{3}-28\right), 3.84(1 \mathrm{H}, \mathrm{brd}, J=9.0 \mathrm{~Hz}, \mathrm{H}-3), 4.05(1 \mathrm{H}, \mathrm{q}$, $\left.J=4.4 \mathrm{~Hz}, \mathrm{H}-4^{\prime}-\mathrm{Nu}\right), 4.27\left(1 \mathrm{H}, \mathrm{dt}, J=7.4,5.5 \mathrm{~Hz}, \mathrm{H}-3^{\prime}-\mathrm{Nu}\right), 4.34(2 \mathrm{H}, \mathrm{m}, \mathrm{H}-$ $\left.5^{\prime}-\mathrm{Nu}\right), 5.29(1 \mathrm{H}, \mathrm{t}$-like, H-12), $5.76(1 \mathrm{H}, \mathrm{d}, J=9.0 \mathrm{~Hz}, \mathrm{NH}), 6.08(1 \mathrm{H}, \mathrm{t}$, $\left.J=6.3 \mathrm{~Hz}, \mathrm{H}-1^{\prime}-\mathrm{Nu}\right), 7.21(1 \mathrm{H}$, br s, H-6-Nu), $8.53(1 \mathrm{H}, \mathrm{br} \mathrm{s}, \mathrm{NH}-\mathrm{Nu})$; MS (negative-API): $m / z 845[\mathrm{M}-1]^{-}(100)$.

General Procedure for Coupling of 3-O-Adipoyloleanolic Acid Derivatives with FK $3000(2 \mathrm{f}, \mathbf{4 f}, \mathbf{6 f})$ A solution of triterpene acid (2a, 4a, 6a, $0.02-0.05 \mathrm{mmmol}$ ), DMAP $(4.0 \mathrm{mg}, 0.03 \mathrm{mmol})$, FK $3000(0.02-0.05$ $\mathrm{mmol})$, and DCC $(0.02-0.05 \mathrm{mmol})$ in $\mathrm{CH}_{2} \mathrm{Cl}_{2}(1 \mathrm{ml})$ was stirred for $10 \mathrm{~h}$ at room temperature. The reaction mixture was treated with water, concentrated to dryness, and purified by column chromatography on RP-18 $\left(\mathrm{H}_{2} \mathrm{O}-\mathrm{MeOH} 3: 7-0: 1\right)$ and silica gel ( $n$-hexane-acetone $\left.9: 1-7: 3\right)$ to obtain pure compounds (2f, $\mathbf{4 f}, \mathbf{6 f})$. It is of interest to note that all the triterpene-FK 3000 conjugates exhibited double signals for the protons and carbons near the nitrogen atom of FK 3000 in the NMR spectra. The phenomenon can be explained as the bold substitution around the nitrogen of FK 3000 resulting in slow conversion between enantiomers at the chiral center of the nitrogen for measurement by ${ }^{1} \mathrm{H}$ - and ${ }^{13} \mathrm{C}-\mathrm{NMR}$. The chemical shift of these signals will be presented as $\delta / \delta$.

$3 \beta$-O-[6-(FK 3000)adipoyl]olean-12-en-28-oic Acid Methyl Ester (2f): $59.3 \%$ in yield; ${ }^{1} \mathrm{H}-\mathrm{NMR}\left(\mathrm{CDCl}_{3}\right) \delta: 0.72(3 \mathrm{H}, \mathrm{s}), 0.83(3 \mathrm{H}, \mathrm{s}), 0.86(3 \mathrm{H}, \mathrm{s})$,
$0.90(3 \mathrm{H}, \mathrm{s}), 0.93(6 \mathrm{H}, \mathrm{s}), 1.13(3 \mathrm{H}, \mathrm{s})\left(7 \times \mathrm{CH}_{3}\right), 2.01\left(3 \mathrm{H}, \mathrm{s}, \mathrm{COOCH}_{3}-6^{\prime \prime}\right)$, $2.04 / 2.05\left(3 \mathrm{H}, \mathrm{s}, \mathrm{COOCH}_{3}-7^{\prime \prime}\right), 3.55 / 3.58\left(3 \mathrm{H}, \mathrm{s}, \mathrm{OCH}_{3}-8^{\prime \prime}\right), 3.62(3 \mathrm{H}, \mathrm{s}$, $\left.\mathrm{COOCH}_{3}-28\right), 3.88\left(3 \mathrm{H}, \mathrm{s}, \mathrm{OCH}_{3}-3\right), 4.49(1 \mathrm{H}, \mathrm{dd}, J=9.0,16.2 \mathrm{~Hz}, \mathrm{H}-3)$, $5.13\left(1 \mathrm{H}, \mathrm{m}, \mathrm{H}-6^{\prime \prime}\right), 5.17(1 \mathrm{H}, \mathrm{br} \mathrm{s}, \mathrm{H}-12), 5.38 / 6.04\left(1 \mathrm{H}, \mathrm{d}, J=4.1 \mathrm{~Hz}, \mathrm{H}-9^{\prime \prime}\right)$, $5.85 / 5.91\left(1 \mathrm{H}, \mathrm{d}, J=3.3 \mathrm{~Hz}, \mathrm{H}-7^{\prime \prime}\right), 6.56 / 6.58\left(1 \mathrm{H}, \mathrm{d}, J=7.0 \mathrm{~Hz}, \mathrm{H}-1^{\prime \prime}\right), 6.74$ $\left(1 \mathrm{H}, \mathrm{d}, J=7.0 \mathrm{~Hz}, \mathrm{H}-2^{\prime \prime}\right)$; MS (negative-API): $m / z 996[\mathrm{M}-1]^{-}(90)$.

$3 \beta$-O-[6-(FK 3000)adipoylimino]olean-12-en-28-oic Acid Methyl Ester (4f): $55.7 \%$ in yield; ${ }^{1} \mathrm{H}-\mathrm{NMR}\left(\mathrm{CDCl}_{3}\right) \delta: 0.76(3 \mathrm{H}, \mathrm{s}), 0.90(3 \mathrm{H}, \mathrm{s}), 0.93$ $(3 \mathrm{H}, \mathrm{s}), 1.02(3 \mathrm{H}, \mathrm{s}), 1.12(6 \mathrm{H}, \mathrm{s}), 1.25(3 \mathrm{H}, \mathrm{s})\left(7 \times \mathrm{CH}_{3}\right), 2.01(3 \mathrm{H}, \mathrm{s}$, $\left.\mathrm{COOCH}_{3}-6^{\prime \prime}\right), 2.04 / 2.05\left(3 \mathrm{H}, \mathrm{s}, \mathrm{COOCH}_{3}-7^{\prime \prime}\right), 3.55 / 3.58\left(3 \mathrm{H}, \mathrm{s}, \mathrm{OCH}_{3}-8^{\prime \prime}\right)$, $3.62\left(3 \mathrm{H}, \mathrm{s}, \mathrm{COOCH}_{3}-28\right), 3.88\left(3 \mathrm{H}, \mathrm{s}, \mathrm{OCH}_{3}-3\right), 5.15\left(1 \mathrm{H}, \mathrm{m}, \mathrm{H}-6^{\prime \prime}\right), 5.28$ $\left(1 \mathrm{H}\right.$, br s, H-12), 5.38/6.04 (1H, d, $\left.J=4.1 \mathrm{~Hz}, \mathrm{H}-9^{\prime \prime}\right), 5.85 / 5.90(1 \mathrm{H}, \mathrm{d}$, $\left.J=2.7 \mathrm{~Hz}, \mathrm{H}-7^{\prime \prime}\right), 6.56 / 6.58\left(1 \mathrm{H}, \mathrm{d}, J=8.0 \mathrm{~Hz}, \mathrm{H}-1^{\prime \prime}\right), 6.74(1 \mathrm{H}, \mathrm{d}, J=8.0 \mathrm{~Hz}$, $\mathrm{H}-2^{\prime \prime}$ ); API-MS (negative): $m / z 1009[\mathrm{M}-1]^{-}(70)$.

$3 \beta$-[6-(FK 3000)adipoylamino]olean-12-en-oic Acid Methyl Ester (6f): $57.2 \%$ in yield; ${ }^{1} \mathrm{H}-\mathrm{NMR}\left(\mathrm{CDCl}_{3}\right) \delta: 0.72(3 \mathrm{H}, \mathrm{s}), 0.77(3 \mathrm{H}, \mathrm{s}), 0.87(3 \mathrm{H}$, s), $0.88(3 \mathrm{H}, \mathrm{s}), 0.90(3 \mathrm{H}, \mathrm{s}), 0.92(3 \mathrm{H}, \mathrm{s}), 1.13(3 \mathrm{H}, \mathrm{s})\left(7 \times \mathrm{CH}_{3}\right), 2.01(3 \mathrm{H}$, $\left.\mathrm{s}, \mathrm{COOCH}_{3}-6^{\prime \prime}\right), 2.04 / 2.05\left(3 \mathrm{H}, \mathrm{s}, \mathrm{COOCH}_{3}-7^{\prime \prime}\right), 3.54 / 3.57\left(3 \mathrm{H}, \mathrm{s}, \mathrm{OCH}_{3}-8^{\prime \prime}\right)$, $3.62\left(3 \mathrm{H}, \mathrm{s}, \mathrm{COOCH}_{3}-28\right), 3.67(1 \mathrm{H}, \mathrm{m}, \mathrm{H}-3), 3.88\left(3 \mathrm{H}, \mathrm{s}, \mathrm{OCH}_{3}-3\right), 5.14$ $\left(1 \mathrm{H}, \mathrm{m}, \mathrm{H}-6^{\prime \prime}\right), 5.27$ (1H, t-like, H-12), 5.38/6.04 (1H, d, $\left.J=3.9 \mathrm{~Hz}, \mathrm{H}-9^{\prime \prime}\right)$, $5.59(1 \mathrm{H}, \mathrm{t}, J=7.9 \mathrm{~Hz}, \mathrm{H}-3), 5.85 / 5.90\left(1 \mathrm{H}, \mathrm{d}, J=2.2 \mathrm{~Hz}, \mathrm{H}-7^{\prime \prime}\right), 6.56 / 6.58$ $\left(1 \mathrm{H}, \mathrm{d}, J=7.2 \mathrm{~Hz}, \mathrm{H}-1^{\prime \prime}\right), 6.74\left(1 \mathrm{H}, \mathrm{d}, J=7.2 \mathrm{~Hz}, \mathrm{H}-2^{\prime \prime}\right)$; API-MS (negative): $\mathrm{m} / \mathrm{z} 995[\mathrm{M}-1]^{-}(100)$.

HIV-1 Protease Assay The same HIV-1 PR assay kit and method were used as described previously. ${ }^{4}$

Anti-HIV Assay MT-4 cells infected with HIV-1 (LAV-1) were used for the antiviral assay as described previously. ${ }^{9)}$

\section{References}

1) Velazquez S., Alvarez R., San-Felix A., Jimeno M. L., Clercq E. D., Balzarini J., Camarase M. J., J. Med. Chem., 38, 1641-1649 (1995).

2) Velazquez S., Tunon V., Jimeno M. L., Chamorro C., Clercq E. D., Balzarini J., Camarase M. J., J. Med. Chem., 42, 5188-5196 (1999).

3) Kimura T., Matsumoto H., Matsuda T., Hamawaki T., Akaji K., Kiso Y., Bioorg. Med. Chem. Lett., 9, 803-806 (1999).

4) Ma C. M., Nakamura N., Miyashiro H., Hattori M., Shimotohno K., Chem. Pharm. Bull., 47, 141-145 (1999).

5) Ma C. M., Nakamura N., Hattori M., Chem. Pharm. Bull., 48, 1681$1688(2000)$.

6) Nawawi A., Ma C. M., Nakamura N., Hattori M., Kurokawa M., Shiraki K., Kashiwaba N., Ono M., Biol. Pharm. Bull., 22, 268-274 (1999).

7) Ohsaki M., Kurokawa M., Nawawi A., Nakamura N., Hattori M., Shiraki K., J. Trad. Med. (in press 2002).

8) Nawawi A., Nakamura N., Meselhy M. R., Hattori M., Kurokawa M., Shiraki K., Kashiwaba N., Ono M., Phytother. Res., 15, 497-500 (2001).

9) Kawahata T., Otake T., Mori H., Morimoto M., Ueba N., Kusumoto I. T., El-Mekkawy S., Hattori M., J. Trad. Med., 13, 59-65 (1996). 